\title{
Preparation of water-soluble asphalt carbon dots and hyperchromic effect to $\mathrm{Cr}(\mathrm{VI})$
}

\author{
Lijuan Gao ${ }^{a}$, Jia Sui , Xiaoqin Qiao, Chaoshuai Hu and Xuefei Zhao ${ }^{b, *}$ \\ Engineering Research Center of Advanced Coal Coking and Efficient Use of Coal Resources, University of Science and Technology Liaoning, \\ Anshan 114051, China
}

\begin{abstract}
Water-soluble asphalt carbon dots (W-CDs) was prepared from medium - temperature coal tar pitch as raw material, via mixed acid oxidation, alkali solubilization and acid precipitation method. It was observed that as-prepared W-CDs were irregular particles with good water dispersibility and the particle size was controlled by the $\mathrm{pH}$ of the acid precipitation solution by a polarizing microscope . Infrared spectroscopy confirmed that the surface of the W-CDs was functionalized by hydrophilic and color-promoting functional groups, which had good water solubility; the thermal weight loss proved to be good thermal stability below $200^{\circ} \mathrm{C}$; the fluorescence spectrum proved that the fluorescence emission peak position of the carbon dots appears red shift with increase of excitation wavelength, with fluorescence conversion property, and its emission light intensity decreases, belonging to non-resonant fluorescence type of direct jump fluorescence; UV spectrum proves that the addition of W-CDs can be chromium (VI) $\mathrm{CrO}_{4}^{2-}$ content The detection limit was increased from milligram to microgram; the molar absorptivity of $\mathrm{CrO}_{4}{ }^{2-}$ was increased by 4 times. The experimentally optimized W-CDs-ultraviolet-viscometry method for measuring $\mathrm{CrO}_{4}{ }^{2-}$ conditions: W-CDs to chromium ratio of $8: 2, \mathrm{pH}$ of 8 , hexamethylenetetramine as a surfactant, and the solution was allowed to stand at room temperature for $45 \mathrm{~min}$.
\end{abstract}

\section{Introduction}

Carbon nanomaterials refer to carbon materials with at least one dimension of less than $100 \mathrm{~nm}$ in the dispersed phase.Carbon quantum dots (CQDs) is a new member of the carbon nanomaterial family. Wang ${ }^{[1]}$ reviewed the application fields and detection methods on CQDs. CQDs as a rising star of carbon nanomaterials, by virtue of their in water solubility, chemical inertness, low toxicity, easy functionalization and high fluorescence intensity, photobleaching resistance, adjustable color of light and other optical properties, have displayed tremendous aemail:gaolijuan@ustl.edu.cn, bemail: zhaoxuefei@163.com momentum in numerous fields such as biosensing, bioimaging, drug delivery, optoelectronics, photovoltaics and photocatalysis, as well as in metal ions, anions, small organic molecules and biomolecules. $\mathrm{Mao}^{[2]}$ used fluorescent CQDs as probes to establish a new method for the determination of $\mathrm{Cu}^{2+}$ based on the fluorescence quenching effect of $\mathrm{Cu}^{2+}$ on carbon quantum dots. $\mathrm{Niu}{ }^{[3]}$ used a bottom-up electrochemical (EC) method to prepare green fluorescent nitrogen-doped carbon quantum dots (N-CQDs), based on the principle of on-off fluorescence (FL), the photoluminescence intensity of N-CQDs is further extended to ON-OFF-ON and ON-OFF-ON-OFF 
by the nature of $\mathrm{Fe}^{3+}$ quenching, used for the detection of $\mathrm{Fe}^{3+}$ in specific environments. Gao ${ }^{[4]}$ produced an on-off-on alternately controlled CQDs fluorescent nanosensor to detect iron $\mathrm{Fe}^{3+}$ in living cells. At the same time, glutathione (GSH) can increase the fluorescence of $\mathrm{CDs} / \mathrm{Fe}^{3+}$ mixed solution, and effectively distinguish cancer cells from normal cells based on the difference in the content of glutathione between cancer cells and normal cells. $\mathrm{Li}^{[5]}$ studied a novel electrochemical sensor for simultaneous determination of $\mathrm{Cd}^{2+}$ and $\mathrm{Pb}^{2+}$ by anodic stripping voltammetry used by NCQDs-GO. In the hybrid system, both N-CQDs and GO have a large electroactive surface area and rich oxygen-containing functional groups, which providing the active site for the compound effectively adsorbs heavy metal ions through electrostatic interaction, improving sensor sensitivity. $\mathrm{Li}^{[6]}$ using CQDs composite $\mathrm{TiO}_{2}$ under visible light reduced $\mathrm{Cr}$ (VI), after 120 min light reaction, the reduction of chromium (VI) rate reached $99.2 \%$, which is the 1.5 times of the reduction efficiency of $\mathrm{TiO}_{2}$. Jiang ${ }^{[7]}$ synthesized $\mathrm{TiO}_{2}$ nanocomposite photocatalysts incorporating CQDs by sol-gel method, CQDs have up-conversion fluorescence characteristics, and the visible light response of $\mathrm{TiO}_{2}$ samples doped with CQDs is significantly improved. $\mathrm{Chen}^{[8]}$ constructed a novel sensor based on $\mathrm{Pt}$ nanoparticle-CQDs/ionic liquid functionalized graphene oxide (PtNPs-CDs/IL-GO) nanocomposites to detect $\mathrm{H}_{2} \mathrm{O}_{2}$. Zhang ${ }^{[9]}$ used CQDs to improve the charge transfer and electrocatalytic properties of the $\mathrm{BiVO}_{4}-\mathrm{SnO}_{2}$ interface. Ye [10] demonstrated that CQDs, as an environmentally friendly photosensitizer, can extend the range of light absorption to the entire visible range significantly. Wang ${ }^{[1]}$ synthesized a novel full-spectrum-driven CQDs (CQDs) $/ \mathrm{Bi}_{2} \mathrm{WO}_{6}(\mathrm{CBW})$ hybrid material to provide full spectrum utilization for enhanced photoactive CQDs heterojunctions in contaminant degradation and energy conversion for reference. Zhang ${ }^{[12]}$ synthesized a $\mathrm{CQDs} / \mathrm{BiPO}_{4}$ nanocomposite photocatalytic material with enhanced visible light absorption and charge separation, the photocatalytic performance of $\mathrm{CQDs} / \mathrm{BiPO}_{4}$ composites is better than that of pure under simulated sunlight or visible light. Hua ${ }^{[13]}$ prepared $\alpha$-BO / N-CQDs composites by low temperature hydrothermal synthesis in one step. When N-CQDs are combined with $\alpha-\mathrm{Bi}_{2} \mathrm{O}_{3}$ nanotubes, the absorbance increases. The introduction of $\mathrm{N}-\mathrm{CQDs}$ can inhibit the photogenerated electron-hole recombination, promote the production of hydroxyl radicals and superoxide radicals, and improve the degradation rate of rhodamine $\mathrm{B}$ by the catalyst under simulated sunlight. Wei ${ }^{[14]}$ prepared $\mathrm{NCDs} / \mathrm{TiO}_{2}$ composites using physical mixing and aging methods. Since NCDs can absorb both ultraviolet light and visible light, the range of light absorption of the material is effectively broadened. In addition, the composite material can effectively prevent the recombination of holes and electrons, and the photocatalytic efficiency is greatly improved. As the content of NCDs in the composite increases, the photocatalytic hydrogen production performance also increases. The recombination of carbon quantum dots and catalyst can not only promote the separation of photogenerated electrons and holes, enhance the utilization efficiency of light by the system, improve the photocatalytic performance, but also enhance the stability of the catalyst ${ }^{[15]}$. The chromium in natural waters is mainly in the valence state of $\mathrm{Cr}$ (III) and $\mathrm{Cr}$ (VI), of which $\mathrm{Cr}(\mathrm{VI})$ is hundreds of times more toxic than $\mathrm{Cr}$ (III) ${ }^{[16]}$. With the continuous development of industry, a large amount of chromium-containing waste is produced in the production process, which directly damages the health and safety of aquatic products and human bodies. Therefore, it is practical to establish a method of detecting $\mathrm{Cr}$ (VI) in water with high density and low detection limit. In this paper, Water-soluble asphalt carbon dots (W-CDs) was prepared from medium - temperature coal tar pitch as raw material, via mixed acid oxidation, alkali solubilization and acid precipitation method. Carbon dots have an up-conversion function of absorbing visible light to emit ultraviolet light and suppressing electron-hole recombination properties, make use of that to enhance the color effect of $\mathrm{Cr}(\mathrm{VI})$, and to establish a method for measuring the trace amount of $\mathrm{CrO}_{4}{ }^{2-}$ by the W-CDs-UV method, the detection limit of $\mathrm{Cr}(\mathrm{VI})\left(\mathrm{CrO}_{4}{ }^{2-}\right)$ content is increased from milligram to microgram.

\section{Experimental section}

\subsection{Preparation of the W-CDs}

Preparation of water-based asphalt carbon dots (W-CDs) 
have obtained under the condition of medium temperature coal tar pitch (Shanxi huazheng company provides, with softening point $83.6^{\circ} \mathrm{C}$ ) with particle size less than $0.2 \mathrm{~mm}$ as carbon source, $7: 3$ (V(sulfuric acid) $/ \mathrm{V}($ nitric acid $)$ ) mixed acid as oxidant; the ratio of carbon source asphalt to mixed acid $1 \mathrm{~g}: 20 \mathrm{~mL}$, Constant temperature $38^{\circ} \mathrm{C}$ for $7.5 \mathrm{~h}$; The following, the contents of the flask were poured into deionized water to terminate the reaction, and place for the night at room temperature; then was filtered using Buchner funnel and washed with water until the filter cake was neutral. The filter cake was transferred to $500 \mathrm{~mL}$ flask, mixed with $1 \mathrm{~mol} / \mathrm{L} \mathrm{NaOH}$ until the $\mathrm{pH}$ of the solution was greater than 12, and stirred at $80^{\circ} \mathrm{C}$ for $1 \mathrm{~h}$ to fully dissolve; and filtered while hot. The filtrate was added with $1 \mathrm{~mol} / \mathrm{L}$ hydrochloric acid to adjust the $\mathrm{pH}$ of the solution to 6 , and vacuum filtered, and the filter cake was recorded as W-CDs 1\#; the filtrate was further precipitated with $1 \mathrm{~mol} / \mathrm{L}$ hydrochloric acid to adjust the $\mathrm{pH}$ of the solution to 4 , and vacuum filtered, and the filter cake was recorded as W-CDs 2\#; the filtrate is further precipitated with $1 \mathrm{~mol} / \mathrm{L}$ hydrochloric acid to adjust the $\mathrm{pH}$ of the solution to 2 , and vacuum filtered, and the filter cake was recorded as W-CDs 3\#.

\subsection{Characteristic Instruments}

The W-CDs were characterized by Fourier transform infrared spectrometer, UV-visible spectrophotometer, fluorescence spectrophotometer, and thermal weight loss tester.

\subsection{Preparation of the W-CDs solution}

Accurate weighing $0.2000 \mathrm{~g}$ the as-prepared W-CDs Put into a $100 \mathrm{~mL}$ bottle, dissolved using $\mathrm{NaOH}$ aqueous solution( $\mathrm{pH} 10$ ), and made up to a volumetric flask of $100 \mathrm{~mL}$ to obtain $2.0 \mathrm{mg} / \mathrm{mL} \mathrm{W}$-CDs solution.

\subsection{Preparation of $\mathrm{CrO}_{4}{ }^{2-}$ solution}

$0.5000 \mathrm{~g} \mathrm{Cr}_{2} \mathrm{O}_{3}$ (analytically pure, Shenyang Dongxing Reagent Factory) solid powder was dissolved in $250 \mathrm{~mL}$ of $1 \mathrm{~mol} / \mathrm{L}$ hydrochloric acid and reacted for $2 \mathrm{~h}$, and the mixture was filtered to remove a trace amount of white solid to obtain $3 \mathrm{mg} / \mathrm{mL} \mathrm{CrO}_{4}{ }^{2-}$ solution.

\section{Results and discussion}

\subsection{Dispersion of the W-CDs}
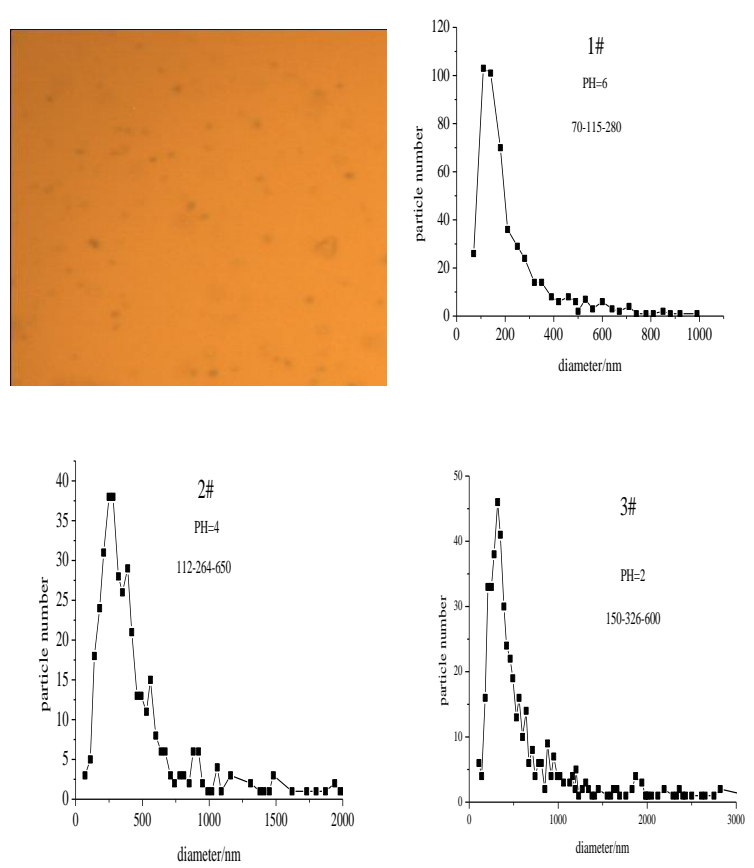

Figure 1. Polarized micrograph and particle size distribution curve of the W-CDs dispersed in water

The morphology of the as-prepared W-CDs were characterized by polarimetric microscope. The polarimetric microscope image (Fig.1) and the corresponding curve of size distribution (Fig.1) illustrate that the W-CDs could be homogeneously dispersed in water solvent, with irregular shape, and hydrodynamic diameters ranging (Fig. 1 (1\#)) from 70 to $280 \mathrm{~nm}$, average size $115 \mathrm{~nm}$. The particle size distribution curve(Fig.1 (1\# 2\# 3\#) ) shows that hydrodynamic diameters of $\mathrm{W}-\mathrm{CDs}$ is related to the $\mathrm{pH}$ value of the solution. The particle size of W-CDs decreases with increasing $\mathrm{pH}$.The $\mathrm{pH}$ of the solution is used to control the size of W-CDs.

\subsection{Characterization of as-prepared W-CDs}

To study the apparent characteristic and optical behavior of the W-CDs, FTIR and UV-vis were applied in detail. FTIR spectroscopy was used to investigate the surface functional groups of W-CDs. As shown in Fig.2, compared with the spectrum of the raw material asphalt, the $\mathrm{W}-\mathrm{CD}$ display new absorption peak, and the intensity 
of the absorption peak change greatly. These peaks are attributed to $\mathrm{O}-\mathrm{H}$ stretching vibration $\left(3610 \mathrm{~cm}^{-1}\right), \mathrm{C}-\mathrm{H}$ stretching vibration $\left(3045 \mathrm{~cm}^{-1}\right), \quad \mathrm{C}=\mathrm{O}$ symmetric stretching vibration $\left(1719 \mathrm{~cm}^{-1}\right), \mathrm{C}=\mathrm{C}$ stretching vibration of aromatic ring skeleton $\left(1593 \mathrm{~cm}^{-1}\right), \mathrm{N}-\mathrm{O}$ symmetry and antisymmetric stretching vibration of the nitro group $\left(1336 \mathrm{~cm}^{-1}\right.$ and $\left.1520 \mathrm{~cm}^{-1}\right)$, O-S-O symmetric stretching vibration of the sulfonic acid group $\left(1042 \mathrm{~cm}^{-1}\right)$. But bending vibration of the alkyl chain of the aromatic ring $\left(735 \mathrm{~cm}^{-1}\right)$ and symmetric stretching vibration $\left(1380 \mathrm{~cm}^{-1}\right)$ disappeared. It can be seen that main types of reactions are oxidation, sulfonation, nitrification, after the medium temperature asphalt treated with mixed acid, and reaction occur in the aromatic ring side chain. more oxygen-containing functional groups whit hydrophilic and facilitated color enhancement function, such as nitro group, hydroxyl group, carboxyl group, sulfonic acid group, are introduced into the surface of the as-prepared W-CDs.

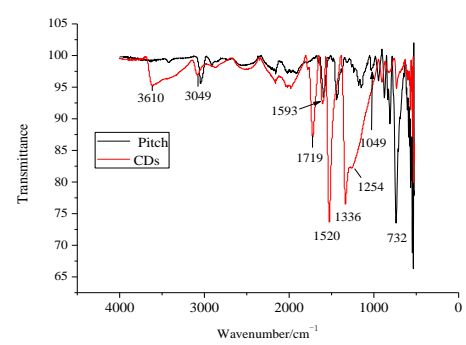

Figure 2. FT-IR spectra of the raw pitch and W-CDs

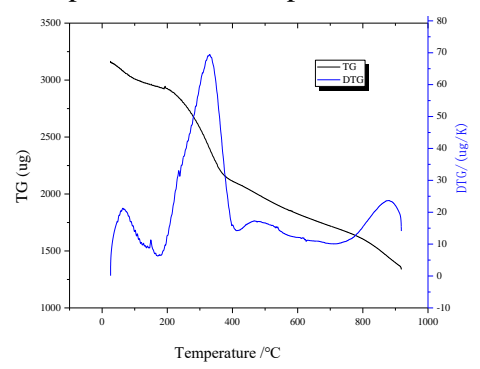

Figure 3. TG/DTG curves of the W-CDs

The TG/DTG curves of the as-prepared W-CDs are presented in Fig.3. As shown in Fig.3, thermal decomposition is clearly revealed. It can be seen from the TG curve that the quality of W-CDs decreases with increasing temperature, and the DTG curve can be roughly divided into three stages according to the image: the first stage, from $36^{\circ} \mathrm{C}$ to $150^{\circ} \mathrm{C}$, are attributed to the evaporation of some small molecules; the second stage, from $200^{\circ} \mathrm{C}$ to $400^{\circ} \mathrm{C}$, are attributed to the pyrolysis of the asphalt original structure functional group; the third stage: from $700^{\circ} \mathrm{C}$ to $900^{\circ} \mathrm{C}$, are attributed to the removal of hydrophilic group in W-CDs. These results show that the temperature of W-CDs be below $200^{\circ} \mathrm{C}$ when applied.
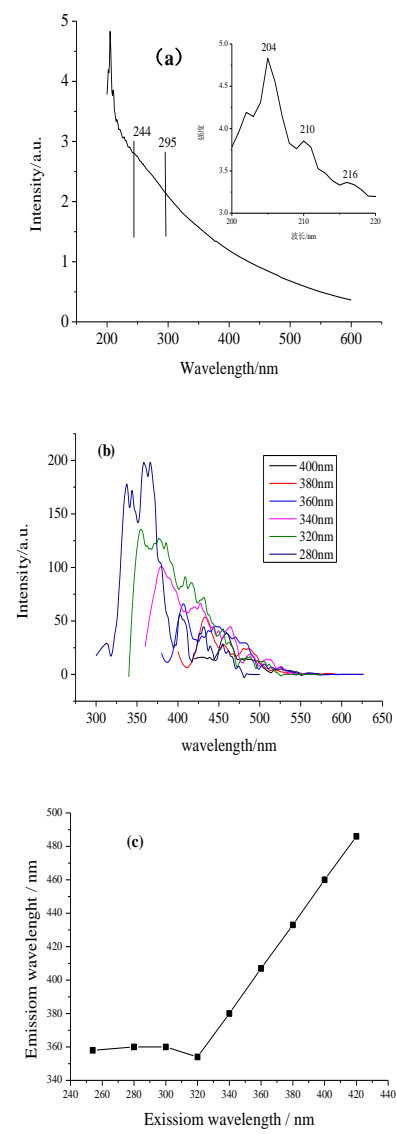

Figure 4. UV-Vis absorption and FL spectra of the W-DCs

As shown in Fig.4(a), the solution of the W-CDs have strong UV-vis absorption peak at $204 \mathrm{~nm}$ and $210 \mathrm{~nm}$, while there is a weak absorption peak at $250 \mathrm{~nm}$, but the absorbance decreases with the increase of absorption wavelength when it is greater than $300 \mathrm{~nm}$, and there is no maximum absorption peak.

The photoluminescence (PL) emission spectra of the W-CDs in the excitation wavelength from $280 \mathrm{~nm}$ to 400 $\mathrm{nm}$ are shown in Fig. 4(b). The position and intensity of the fluorescence emission peak of the W-CDs are related to the excitation wavelength. The emission peak of the W-CDs appears the red-shifted in position and reduced intensity whit the excitation wavelength increases. The excitation avelength, which results in emission spectrum absorption peak is the strongest, is $280 \mathrm{~nm}$, meanwhile the emission wavelength is $360 \mathrm{~nm}$. Which proves that W-CDs has fluorescence conversion properties, It belongs to the non-resonant fluorescence type of direct jump fluorescence. In order to investigate the 
photoluminescence characteristics of W-CDs, especially the dependence on excitation light, excitation spectra of W-CDs in excitation wavelength from $254 \mathrm{~nm}$ to $420 \mathrm{~nm}$ are shown in Fig. 4(c). When the excitation wavelength is increased from $254 \mathrm{~nm}$ to $320 \mathrm{~nm}$, it can be seen that the maximum emission wavelength is almost constant, meanwhile the intensity gradually heighten; When the excitation wavelength is increased from $320 \mathrm{~nm}$ to 420 $\mathrm{nm}$, it can be seen that the maximum emission wavelength is red-shifted, meanwhile the intensity gradually decreases.

From the theoretical analysis of the surface state, it is considered that the carbon quantum dots are surface-defective luminescence. When the surface electrons of the carbon quantum dots are excited by ultraviolet light, electron-hole pairs are formed, and electrons are instantaneously captured by surface defects during the migration process, thereby generating fluorescence. Meanwhile, a photon with adequate energy can excite an electron in the HOMO level of CQDs, transferring the excited electron to the LUMO level of CQDs. The emission from CQDs shifted to higher wavelength as the intensity increases, which is characteristic of surfacedefect emission derived from CQDs as reported by others. which may arise from the $\pi-\pi *$ transition of the nanocarbon particles and from the excitation energy sink of the surface states.

\subsection{Effect of the W-CDs on $\mathrm{CrO}_{4}{ }^{2-}$ molar absorption coefficient}

Figure 5 (a) compares the UV-Vis absorption spectra of $\mathrm{CrO}_{4}{ }^{2-}$ and the as-prepared W-CDs. As shown in Fig. 5(a), it was found that the maximum absorption peaks appear at $248 \mathrm{~nm}, 280 \mathrm{~nm}$ and $350 \mathrm{~nm}$ for the $\mathrm{CrO}_{4}{ }^{2-}$ solution, and the absorption intensity decreases in turn; the maximum absorption peak appears less $250 \mathrm{~nm}$ for the as-prepared W-CDs suspension liquid, and no maximum absorption peak appears as wavelength larger $250 \mathrm{~nm}$. The as-prepared W-CDs suspension liquid, at $350 \mathrm{~nm}$, has almost no absorption; but the $\mathrm{CrO}_{4}{ }^{2-}$ solution has a weak maximum absorption. In order to reduce the influence of the absorption of the the W-CDs itself, the measurement wavelength of $\mathrm{CrO}_{4}{ }^{2-}$ is selected to be 350 nm.
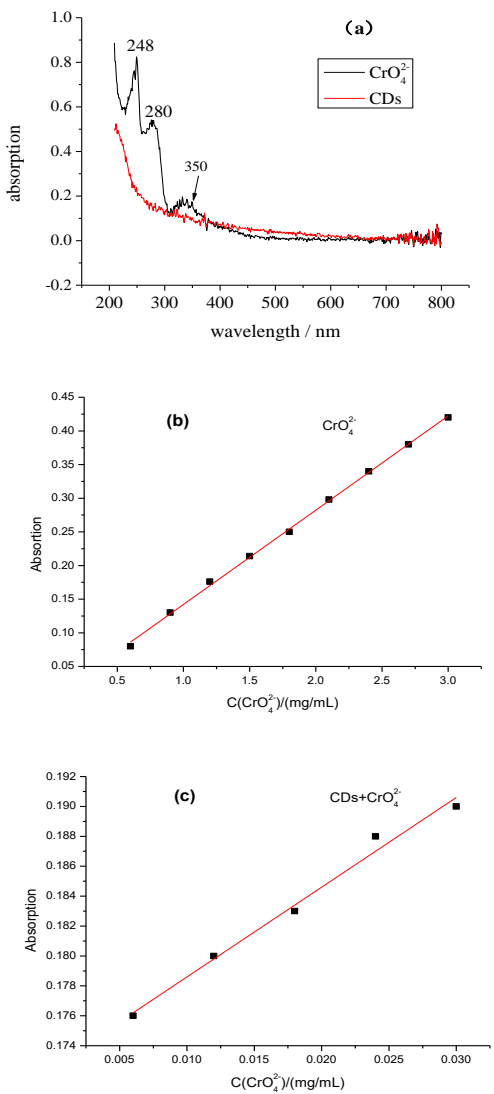

Figure 5. Effect of the as-prepared W-CDs on $\mathrm{CrO}_{4}{ }^{2-}$ absorption coefficient

Figure 5 (b) shows the linear correlation of the absorbance of the $\mathrm{CrO}_{4}{ }^{2-}$ solution at wavelength of 350 $\mathrm{nm}$, and Figure 5 (c) shows the linear correlation of the absorbance at wavelength of $350 \mathrm{~nm}$ for a $\mathrm{CrO}_{4}{ }^{2-}$ solution with $6 \mathrm{mg} / \mathrm{L}$ the as-prepared W-CDs. It can be seen from Fig. 5 that the pure $\mathrm{CrO}_{4}{ }^{2-}$ solution can measure the linear range of ultraviolet absorption from $0.6 \mathrm{mg} / \mathrm{mL}$ to 3.0 $\mathrm{mg} / \mathrm{mL}$, and the linear linear regression equation: $\mathrm{A}=0.00202+0.140(\mathrm{C} /(\mathrm{mg} / \mathrm{mL}))$, correlation coefficient $\mathrm{R}=0.9995$; With the addition of the W-CDs, the $\mathrm{CrO}_{4}{ }^{2-}$ solution can measure the linear range of UV absorption from $0.006 \mathrm{mg} / \mathrm{mL}$ to $0.030 \mathrm{mg} / \mathrm{mL}$. The linear regression equation: $\mathrm{A}=0.1726+0.600 \quad(\mathrm{C} /(\mathrm{mg} / \mathrm{mL}))$, correlation coefficient $\mathrm{R}=0.9940$.

According to Beer's law, $\mathrm{A}=-\log \left(\mathrm{I} / \mathrm{I}_{0}\right)=-\lg \mathrm{T}=\varepsilon \mathrm{c}$; where A: absorbance, $I_{0}$, I: intensity of incident light and transmitted light, $\mathrm{T}$ : transmittance, $\varepsilon$ : molar absorption coefficient of sample, c: sample concentration. It can be seen from Fig. 5 that, added to the W-CDs , a smaller concentration of $\mathrm{CrO}_{4}{ }^{2-}$ can be measured accurately. The measured value of concentration (c) becomes smaller, and 
the corresponding value of absorbance (A) increases. According to Beer's law, $\varepsilon$ increases from 0.140 to 0.600 . Generally, $\varepsilon$ becomes larger, indicating that the absorption band of the measured substance becomes stronger, which is favorable for the meas urement of the absorbance, and it can be seen that the W-CDs can enhance the $\mathrm{CrO}_{4}^{2-}$ molar absorption coefficient.

\subsection{Optimization of conditions for measuring $\mathrm{CrO}_{4}{ }^{2-}$ by W-CDs-UV-vis spectrophotometry}

To optimize the conditions for measuring $\mathrm{CrO}_{4}^{2-}$ by W-CDs-UV-visible spectrophotometry, influence factors such as temperature, ratio of $\mathrm{W}-\mathrm{CDs}$ to $\mathrm{CrO}_{4}{ }^{2-}$, time, $\mathrm{pH}$, surfactant and other factors were investigated by experiment.

The solution for the experiment were prepared .The $\mathrm{CrO}_{4}{ }^{2-}$ solution $(3 \mathrm{mg} / \mathrm{mL})$ were mixed with the $\mathrm{W}-\mathrm{CDs}$ solution $(0.020 \mathrm{mg} / \mathrm{mL})$ according to different volume ratios, and the absorbance was measured at 360nm. The effect of the volume ratio of the $\mathrm{W}-\mathrm{CD}$ to $\mathrm{CrO}_{4}{ }^{2-}$ on the absorbance of the test solution is shown in Fig. 6(a), as the proportion of the $\mathrm{W}-\mathrm{CDs}$ to $\mathrm{CrO}_{4}{ }^{2-}$ increases, the absorbance increases, and reaches the maximum at $\mathrm{V}(\mathrm{CDs}): \mathrm{V}\left(\mathrm{CrO}_{4}{ }^{2-}\right)=8: 2$, so the best W-CDs to $\mathrm{CrO}_{4}{ }^{2-}$ is 8:2. Fig. 6(b) shows the effect of time and temperature on the absorbance of the test solution. Under optimized W-CDs to $\mathrm{CrO}_{4}{ }^{2-}$ ratio $8: 2$, the test solution were heated to $30{ }^{\circ} \mathrm{C}, 45{ }^{\circ} \mathrm{C}, 60{ }^{\circ} \mathrm{C}, 75{ }^{\circ} \mathrm{C}, 90{ }^{\circ} \mathrm{C}$ respectively, and measured the absorbance at $0 \mathrm{~min}, 15 \mathrm{~min}, 30 \mathrm{~min}, 45$ min, $60 \mathrm{~min}$, the results are shown in Fig. 6 (b). It can be seen from Fig. 6(b) that the absorbance at different temperatures increases first and then decreases with time, and the maximum value appears at $45 \mathrm{~min}$, so the optimal time is $45 \mathrm{~min}$. The maximum absorbance decreases with the increasing temperature, so the room temperature $30^{\circ} \mathrm{C}$ is the optimum temperature.

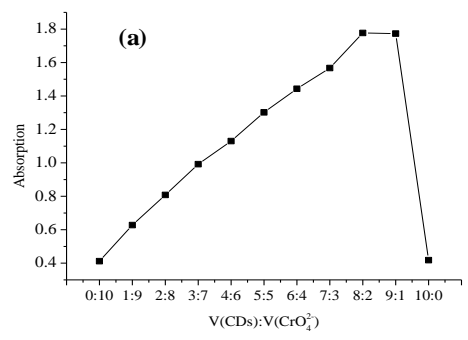

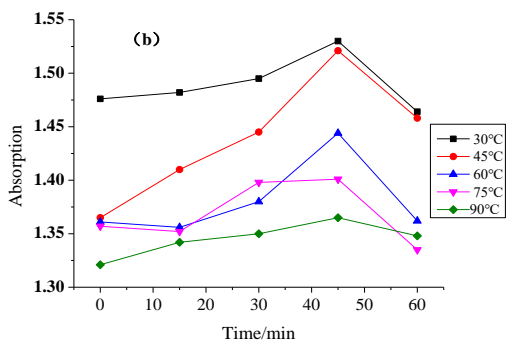
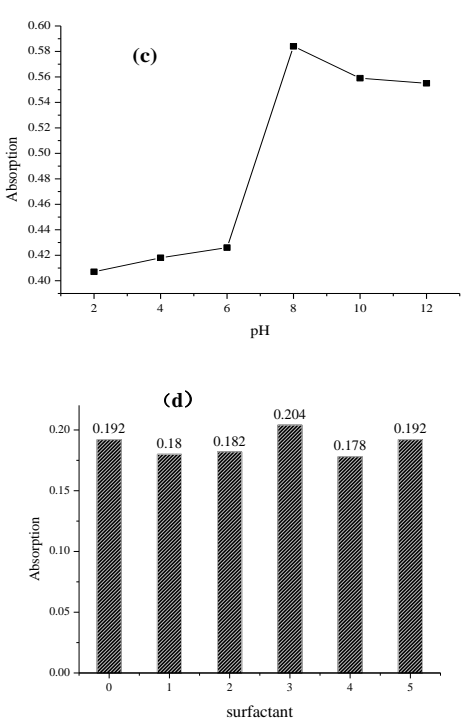

Figure 6. Conditions for measuring $\mathrm{CrO}_{4}{ }^{2-}$ by W-CDs - UVvis spectrophotometry

Fig. 6 (c) shows the effect of $\mathrm{pH}$ on the absorbance of the test solution. Six solutions whit W-CDs to $\mathrm{CrO}_{4}{ }^{2-}$ ratio $8: 2$, adjusted the $\mathrm{pH}$ values to $2,4,6,8,10,12$ respectively, and measured their absorbance, the results are shown in Fig. 6(c). According to Fig. 6(c), as the $\mathrm{pH}$ value of the solution increases, the value of the absorbance increases first gradually and then decreases when $\mathrm{pH}$ reaches 8 , the absorbance is maximum, so the selected $\mathrm{pH}$ value is 8 .

Fig.6 (d) presents the effect of the addition of surfactant on the absorbance of the test solution. X-axis indicate selected surfactants (0 (no surfactant), 1(polyacr-ylamide), 2 (sodium hydrogen phthalate), 3(hexameth-ylenetetramine), 4 (adipate), 5 ( polyethylene glycol)). Compared with the non-surfactant mixture, the absorbance increases when No. 3 surfactant (hexamethylenetetramine) added, and other surfactants have no significant effect. Therefore, hexamethylenetetramine is selected as the added surfactant.

\section{Conclusion}


(1) Carbon dots with hydrophilic and color-promoting functional groups on the surface can be prepared by the water-insoluble medium-temperature asphalt as raw material, via mixed acid oxidation, alkali solubilization and acid precipitation method; the particle size of the carbon dots can be controlled by the $\mathrm{pH}$ value of the acid precipitation solution, and the particle size distribution range is $70 \mathrm{~nm}$ to $280 \mathrm{~nm}$ at the $\mathrm{pH}$ value of 6 , with a mean value of $115 \mathrm{~nm}$, the carbon dots are irregular particles and disperse well in water.

(2) When the particle size of the as-prepared carbon dots is larger than $300 \mathrm{~nm}$, the UV absorbance decreases with the increase of the absorption wavelength, and there is no maximum absorption peak; and the fluorescence emission peak red-shifted with the increase of the excitation wavelength, which has fluorescence conversion property; emission intensity reduced, which is a direct linear fluorescence of non-resonant fluorescent type. The addition of as-prepared W-CDs can increase the UV molar absorption coefficient of $\mathrm{CrO}_{4}{ }^{2-}$ by 4 times, and the density of detection increases from $\mathrm{mg}$ level to ug level.

(3) Optimization of conditions for measuring $\mathrm{CrO}_{4}{ }^{2-}$ by as-prepared W-CDs-visible spectrophotometry : W-CDs $(0.020 \mathrm{mg} / \mathrm{mL})$ to $\mathrm{CrO}_{4}{ }^{2-}(3 \mathrm{mg} / \mathrm{mL})$ volume ratio $8: 2$, the $\mathrm{pH} 8$, and the hexamethylenetetramine surfactant, the solution is allowed to stand at room temperature for 45 min.

\section{Acknowledgements}

This work was supported by the National Natural Science Foundation of China (U1361126), the Specialized Research Fund for the Doctoral Program of Higher Education (20132120110001).

\section{References}

[1] R. Wang, K.Q. Lu, Z.R. Tang , Y.J. Xu. J. Mater Chem A, 5, 3717-3734 (2017)

[2] X.J. Mao , H.Z. Zheng, Y.J. Long. Journal of Southwest University ( $\mathrm{N}$ atural Science Edition), 32(9): 40-43 (2010)

[3] F.S. Niu , Y.L. Ying , X. Hua , Y.S. Niu , Y.H. Xu , Yi-.T. Long. Carbon, 127，340-348(2018)

[4] G. Gao, Y.W. Jiang , H.R. Jia , J.J. Yang, F.G. Wu . Carbon, 134， 232-243(2018)
[5] L.b. Li, D. Liu, A.P. Shi , T.Y. You. Sens. Actuators, B $255,1762-1770(2018)$

[6] Y.R. Li, Z.M. Liu, Y.C. Wu, J.T. Chen, J.Y. Zhao, F.M. Jin, P. Na. Appl. Catal., B: Envir. 224, 508 -517(2018)

[7] T.T. Jiang, Y. Shi， J. Ke， K.l.

Xu. J. NU. (Nat. Sci.)， 39（1）, 183-142（2018）

[8] D.D. Chen , X.M. Zhuang , J. Zhai , Y.Yyun. Zheng, H. Lu , L.X. Chen . Sens. Actuators, B , 255 , 1500 $-1506(2018)$

[9] Z.M. Zhang , X. Jiang , J.F. Mei , Y.X. Li , W.H. Han , M.Z. Xie, F.C. Wang , E.Q. Xie. Chem. Eng. J. $331,48-53(2018)$

[10] K.H. Ye, Z.L. Wang, J.W. Gu, S. Xiao, Y.F. Yuan, Y.Zhu, Y.M. Zhang, W.J. Mai and S.H.

Yang. Energy Envir. Sci, 10, 772-779 (2017)

[11] J.J. Wang , L. Tang , G.M. Zeng , Y.C. Deng , H.R. Dong, Y.N. Liu , L.L. Wang , B. Peng, C. Zhang, F. Chen. Appl. Catal., B: Envir , 222,115-123(2018)

[12] Z.J. Zhang, J.Y. Xu, H.B. Zeng, N. Zhang. J J. Inorg. Mater, 33 ( 5),582-586 (2018)

[13] C.H. Hua, H.C. Ma, X.L. Dong, X.F. Zhang. Chem. J. Chin. Univ, 39(2):200-205 (2018)

[14] J. Wei, X.D. Li, H.Z. Wang, Q.H. Zhang, Y.G. Li. J. Inorg. Mater , 30(9):925-930 (2015)

[15] J.Q. Pan, Y.Z. Sheng, J.X. Zhang, J.M. Wei, P. Huang, X. Zhang , B.X. Feng . J. Mater. Chem. A, 2, 18082-18086 (2014)

[16] H.R. Wu， L. Jiang， J. Wu， S.N. Li， R.Cheng, H.Y. MA. Guangzhou Chem. Ind， 46 ( 5):50-52（2018） 